\title{
A Cross-Cultural Evaluation Framework for Behavioral Biometric User Authentication *
}

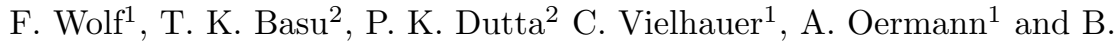 \\ Yegnanarayana $^{3}$ \\ 1 Otto-von-Guericke University Magdeburg \\ at Magdeburg, 39106 Magdeburg, Germany \\ 2 Indian Institute of Technology, Kharagpur \\ at Kharagpur 721302, India \\ 3 Indian Institute of Technology, Madras \\ at Chennai, India
}

\begin{abstract}
Today biometric techniques are based either on passive (e.g. IrisScan, Face) or active methods (e.g. voice and handwriting). In our work we focus on evaluation of the latter. These methods, also described as behavioral Biometric, are characterized by a trait that is learnt and acquired over time. Several approaches for user authentication have been published, but today they have not yet been evaluated under cultural aspects such as language, script and personal background of users. Especially for voice and handwriting such cultural aspects can lead to a significant and essential outcome, as different spoken and written languages are being used and also the script used for handwriting is different in nature.
\end{abstract}

\section{Motivation}

The goal of our work is to analyze cross-cultural aspects of handwriting data as a digital input for biometric user authentication. Therefore, we have designed and developed a biometric evaluation framework within the CultureTech project which focuses on cultural impacts to technology in an EuropeanIndian cross-cultural context. The framework, its methodology as well as a short outline of evaluation aspects have already been presented in Schimke et al. (2004). In this paper we will enhance evaluation aspects into detail and derive first hypotheses of the correctness and usability of biometric user authentication systems for different cultures.

Evaluation aspects, considered to be analyzed, are formulated as two different but related sources. First, so called meta data is collected. A taxonomy for meta data is presented in Vielhauer et al. (2005). Following this taxonomy, meta data is differentiated in two main categories, the technical and the nontechnical one. While the technical meta data implies hardware and software

\footnotetext{
* This publication has been produced with the assistance of the European Union (project CultureTech, see http://amsl-smb.cs.unimagdeburg. de/culturetech/). The content of this publication is the sole responsibility of the University Magdeburg and their co-authors and can in no way be taken to reflect the views of the European Union.
} 
parameters such as the device identification (tablet-based or pen-based), the non-technical meta data addresses the cultural and personal background of a person. In Jain (2004a) and Jain (2004b) non-technical meta data is presented as soft biometrics. Non-technical meta data are subject of our research and focus of this paper. We introduce a new classification of meta data in order to reach two distinct goals: Given the fact, that personal information like age and gender can be statistically estimated by analyzing human handwriting Tomai et al. (2004), the first evaluation goal is the derivation of cultural characteristics of a person such as ethnicity, education, and language by statistical or analytical means of handwriting dynamics. The second goal is to evaluate the impact, which certain meta data can have for biometric user authentication systems based on handwriting, especially if additional facts of the personal background like culture, spoken and written languages, ethnicity influence biometric handwriting user authentication processes in order to estimate the accuracy. In this paper we focus on the latter.

As the second evaluation aspect, biometric handwriting data and the related non-technical meta data are analyzed to estimate how the condition of a person during the process of experimental testing influences the behavioral biometric data. In this context, additional incidents as a special class of meta data can influence biometric handwriting data in certain ways. Beside others, these incidents are determined through cross-cultural experiences of the person in the far or near past, i.e. he or she stayed abroad, the person's familiarity with given tasks like the person's familiarity with the hardware such as the digitizer tablet and pen and the attitude towards digital biometric systems in general. Hence, this class of meta data has to be specified and analyzed to adapt the recognition or authentication algorithms in order to enhance their performance and quality measured by the Equal Error Rate (EER). To read more about EER we refer to Scheidat (2005) and Vielhauer (2006).

Considering non-technical meta data and the cross-cultural context, our methodology is as follows: In order to evaluate the process of user authentication in bilingual or multilingual environment, handwriting data is collected in three different countries, India, Italy and Germany. Based on this, we focus on developing hypotheses based on behavioral biometric handwriting input and the collected meta data. As Vielhauer (2006) will show, meta data can have an essential impact in order to achieve more reliable and correct results in biometric user authentication systems for handwriting. In this paper hypotheses are derived by analyzing biometric handwriting data and the subject related meta data. These hypotheses not only address particular, a biometric system influencing factors, but also evaluate them.

Our framework will be of relevance in two main areas of the cross-cultural biometric field. First, it is a new evaluation system for biometric user authentication in bilingual or multilingual environment and second, our system can be used for user verification in a cross-cultural context. 
The paper is structured as follows: In section 2, the process of data collection is introduced and the experimental framework is briefly outlined. In section 3, an enhanced classification of meta data is presented. This is followed by the description of the evaluation methodology and the formulation of hypotheses in section 4. First results which are based on an experimental data collected in Germany are presented in section 5. Finally, section 6 concludes by summarizing the paper and providing a perspective on future work.

\section{Meta data - Definition}

As briefly mentioned in our introduction, the overall definition of meta data needs to be specified and classified. There exist different, but closely related classifications of meta data as Figure 1 illustrates.

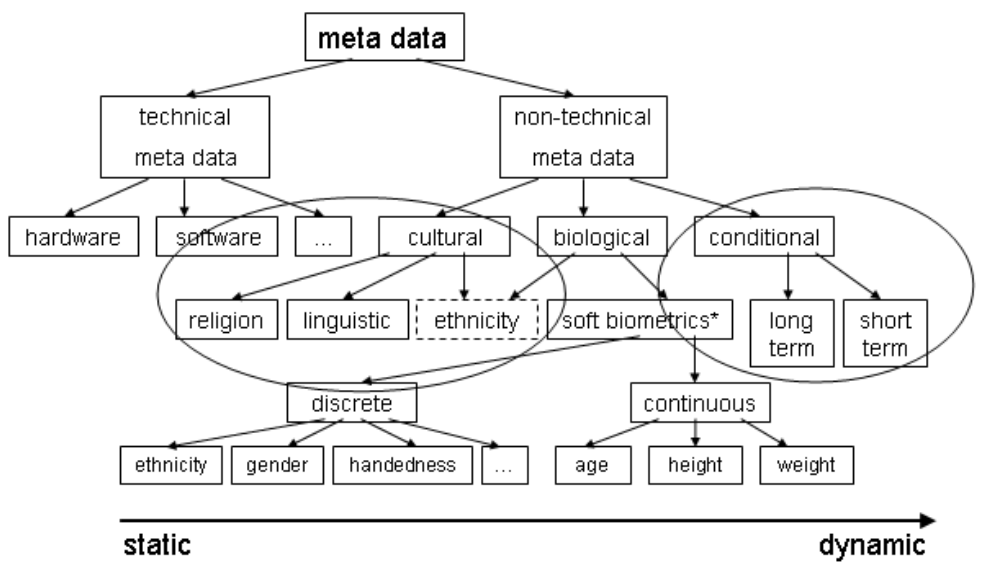

Fig. 1. The meta data hierarchy

A basic meta data taxonomy is presented in Viehauer et al. (2005) and differentiates technical and non-technical meta data. Technical meta data include aspects of the used device such as hardware and software specifications. For handwriting sampling technical meta data specify the digitizer tablet and the used pen as well as the used framework. There exist three classes of non-technical meta data. One class includes aspects of biological meta data. Those meta data, described in Jain (2004a) and Jain (2004b) as soft biometric traits, are continuous or discrete parameters, which provide some information about the individuals biological background. Discrete parameters are of a certain static characteristic such as ethnicity, gender, and handedness, while continuous parameters are more dynamic such as age, 
weight, and height. Ethnicity refers to the second class of non-technical meta data - the cultural class, which joins religious, linguistic and ethnic aspects as almost static parameters. The third class of non-technical meta data is determined though dynamic, conditional parameters of the person. This class is divided in the long term and the more dynamic short term conditional meta data. In this paper we focus on the cultural and conditional, non-technical meta data. Biological parameters like year of birth, ethnicity, gender, and handedness never change and are valid for one specific person, whenever the data collection may take place. While Jain (2004a) and Jain (2004b) use these biological parameters to limit the group of subjects, a biometric authentication process is used on, our goal is to investigate, to which extend the meta data influences the biometric data during collection.

The reason for meta data being a major focus of recent research in the field of biometric user authentication is their impact to improve the performance of traditional biometric systems. In our investigations we establish a double tracked procedure. In order to improve the accuracy and reliability of algorithms for biometric handwriting user authentication systems, we analyze static, as well as dynamic parameters of meta data. Static meta data of the cultural background of a person is collected at the beginning of a sample enrollment, and it is stored as a profile in a data base. Once being collected, this meta data is valid for all upcoming tests, concerning the specified subject. Dynamic meta data of the conditional background of a person is collected through a questionnaire before and after the enrollment. This meta data includes the experiences of a person, which have been gained during his or her biographic past. As mentioned before, these conditional aspects are differentiated in long term and short term parameters. The long term parameters are gained through long term experiences, for example staying abroad (e.g. learning new languages, writing standards), working habits (e.g. familiarity with computers and technological systems), and educational specialization (e.g. knowledge of biometric systems, attitudes). These dynamic parameters can change over time. New experiences can be made, old experiences can be forgotten. Short term conditional parameters have a very dynamic characteristic. They can be applied exclusively while data enrollment, since they describe the persons actually condition during recording: does he or she feels tired, nervous, or stressed etc.. These parameters significantly change over time.

Both classes of meta data, the cultural, as well as the conditional, essentially influence the output of the sample class collection. Our aim is to analyze their impact in order to improve biometric user authentication systems for handwriting. 


\section{Data Collection and Experimental Framework}

In this section we briefly present the environmental and technical concept of the system as described in Schimke et al. (2004). This includes the data collection and the description of the experimental framework. The structure of all components such as handwriting recording, meta data, and conditional information will be described.

Our framework contains a generic system design considering additional meta data models and an audio capability and consists of the following components:

- Sample tasks: The subjects are asked to write 48 given writing samples, each is to repeat 10 times. The different samples are available in English and German.

a) Traditional handwriting tasks like giving signature.

b) Words / sentences (statements and questions) of different complexity

c) Numbers

d) Questions about the name, heritage and age are to answer.

- Data Recorder: Implements the A/D conversion from handwriting and audio sampling devices. For sampling, we use tablet PC hardware, equipped with active pen-based (WinTab compatible) digitizer hardware and onboard audio device.

- Evaluation Database: Stores the complete audio and handwriting signals along with synchronized meta data, non technical (once stored for each subject) and technical (stored after each sample).

- Test Controller: Reproduces user inputs in batch mode process. The operational sequence of batch runs is defined by Test Profiles, which feed reproduced signals from the Evaluation Database to plug-in Algorithms in order to be evaluated, and protocol their results to the test log.

- Questionnaire: Independent of the system. Questions are about:

a.) long term

b.) short term

Further, we define a test module as a specified set of handwriting or audio recordings of one person in one language. As described before, there are different types of recordings: questions to answer, words, numbers and sentences to write. A test session is a set of test modules of one person. A hole test session can be set up as follows:

1. Collection of the meta data of the subject

2. Recording of the test modules

3. Filling in the questionnaire

During our recordings most of the subjects have been invited to two or three test sessions: First, handwriting data in their native language was collected, second, handwriting data in a second language (usually English) was 
collected and third, audio data in their native language was collected. It is planned to add another test session: a bi- or multi-lingual speech recording session.

\section{Methodology - First Hypotheses}

In our test modules, data of 32 persons has been enrolled. During these experiments, certain meta data could be categorized and specified as follows:

Technical meta data concerning the aspects of recording and environment:

- Data recorded in a laboratorial environment

- Test modules all the same (48 samples, 10 repetitions each)

- Time of recording: between 10AM - 5PM

- Tool for handwriting recording: software PlataSign and digitizer tablet

Non-technical meta data that can be assumed as true for all registered subjects of the test modules:

- Educational background: Academical (recordings took place at University)

- A-levels

- Native Language: German

- Learned Languages: English

- Learned languages: at least 1 (English), Maximum of 3

- Scripts: Maximum of 2

- Age between 19 and 30

- Subjects handedness: right ${ }^{1}$

- Subjects religion: Christian (protestant) or no religion

- Equality of gender representation

By analyzing the questionnaires, a high motivation and willingness of the subjects could be observed.

The goal of our work is to propose a set of hypotheses considering the static and dynamic classes of meta data, as well as multi cultural aspects of user authentication. Thus, we want to demonstrate and evaluate, that additional information such as meta data can improve the performance of behavioral biometric user authentication systems. Our focus is on handwriting.

Based on the collected handwriting data, meta data, and the questionnaires, our hypotheses are structured as follows: A variety of hypotheses, which initiatively concerns only visual noticeable features, can be derived from the collected data. In this paper, we have chosen hypotheses concerning two of the most obvious aspects and parameters, which have been collected. First, hypotheses about the difference of handwriting will be outlined,

\footnotetext{
${ }^{1}$ Rigid reeducation of left- to right-handedness in the former GDR [6]
} 
depending on used languages. Second, gender-specifications and familiarity with different languages will be analyzed.

The major goal of this evaluation is to group different handwriting characteristics together. Hence, particular handwriting sample features can be clustered. Therefore, we introduce our methodology by analyzing certain groups of test values, as it is illustrated in Figure 2. A subject's input, which consists of conditional and cultural meta data as well as recorded biometric data, result in certain test values or test module parameters. Test values are being analyzed by two different aspects, syntactical and semantical. Thus, hypotheses can be derived and retrospectively crosschecked with the input.

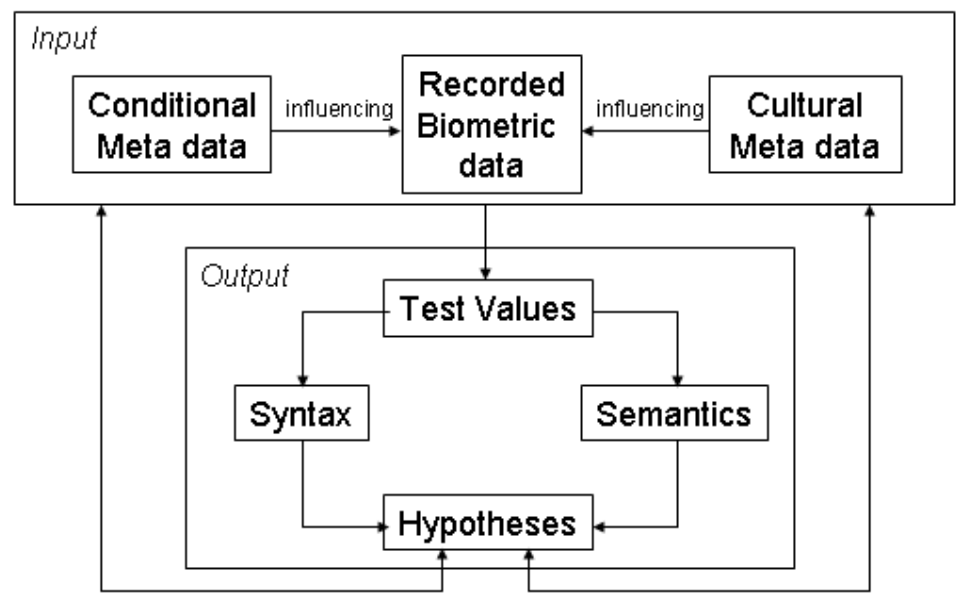

Fig. 2. Scheme of Methodology

Our methodology has the following structure:

a.) Analyzing by syntactical aspects. The syntax is the physical entity of the handwriting samples. It contains:

- test module parameters (SampleID, timestamp),

- dynamic writing features (e.g. velocity, pressure and the pen's angles, in particular, altitude and azimuth),

- writing features (e.g. position of points, tilt angle, gabs, horizontal and vertical dimensions, length of lines).

b.) Analyzing by semantic aspects. The semantic describes aspects of the content and can be derived from syntactical features. It contains:

- personal and special meanings of free chosen answers to questions (e.g. forename and surname, symbols, passphrase),

- added, elided or twisted aspects of words (characters, upper/lower case) and sentences (words, characters, question/exclamation marks). 
and the appearance of test modules such as:

- readability of text

- peculiarity (simplification and enrichment, in general)

- variability (accessory of simplification and enrichment during variation in time or at special combination of characters)

c.) Meta-data have been analyzed and investigated considering special aspects at a) and b), in particular, with regard to gender and languages.

d.) Questionnaires have been analyzed and investigated considering special aspects at a) and b), in particular, with regard to acceptance, stays abroad and familiarity with computers.

e.) Developing of hypotheses, which group aspects of a \& c, a \& d, b \& c and b \& d. Obviously, the connections from syntactical or semantic aspects to meta data or the questionnaire are analyzed separately performing 1:1 relationships.

Further steps are under recent investigations.

f.) Evaluation of the hypotheses formulated at e).

g.) Combining of conclusions via evaluation crossing (developing 1:2 relationships). Including aspects of cross-linguistic influence (as a Germanspeaker writing English).

h.) Analyzing Indian and Italian data (a)-h)) and Indians and Italians writing English or German.

i.) Combining conclusions cross-culturally of all test modules (Indian, Italian, German) and combining tests with speech-authentication. This includes aspects of native and non-native speaker, different scripts, religions, cross-linguistic influence.

\section{Results}

The following hypotheses can be summarized:

Influences on numbers, based on stays abroad: Independent of other meta data as gender, attitude or age a high semantic variability of the numbers "1", "7" and "9" could be mentioned. Some of the subjects have a very similar writing style. The used style lacked features of the expected "German standard" 2 and was alike the "English standard" 3 . These observations are supported by the questionnaires, which indicate the subject's stays abroad experiences. It could be noticed, that most of the subjects, who stayed abroad (in English-speaking countries) still use the English standards, but hardly anyone who hasn't been abroad did. But the more it was dated back, the more subjects re-adapted to the German standard again. Staying abroad also

\footnotetext{
2 "1" having a slight up stroke with a little stroke on top, 7 including a cross bar in the middle, "9" having a curvature under the upper circle

3 "1" just having a light up stroke, 7 lacking the cross bar, "9" having a stroke instead of a curvature
} 
influenced choices of individual samples (English phrases more used than in the non-stay abroad group), and orthography (less upper case used).

Influences of gender on syntactical features: The analysis of male subjects test modules compared with female subjects showed, that men had much higher writing pressure than the women. But the female pressure had a higher variability than the male did. In average, the male subjects used tilt angles (0-30) and were much lower than the female subjects ones (30-100). The horizontal and vertical dimensions also varied.

Influences of gender on semantical features: In average, the male subjects writing variability was much higher than the female subjects. Male subjects tended to use interpret much more than the female subjects, especially at sentences. Words were twisted, displaced or left out, question and exclamation marks added consciously much more often, than in the female group. Another difference was mentioned concerning the perfectionism and patience. Where men where contend with the visual appearance of their samples, even if mistakes were made, and tended to finish as soon as possible, women were adamant to correct their work much more often.

Besides these hypotheses, more hypotheses were formulated, concerning attitudes (willingness to give information vs. knowledge of biometric systems), influences of used soft- and hardware (distracting software warnings, hardware failures) and relationships of subjects to the supervisor (befriended or unknown) just to name a few. But was mentioned before we restrict to hypotheses considering the most obvious parameters.

\section{Conclusions \& Future Work}

In order to investigate and recognize differences between cultures (here India, Italy, Germany) and languages, we have introduced a new approach to formulate hypotheses concerning the impact of certain meta data (cultural and biological background) and conditions (experiences and attitudes) on behavioral biometric data, focussing handwriting data. By evaluating our hypotheses a new area of research in the field of inter- and intra-cultural, as well as multi-modal user interfaces will be opened. Especially for behavioral biometric authentication systems more accurate and reliable results and higher security levels against forgery may be accomplished. 


\section{References}

JAIN, A. K. et al. (2004): Soft Biometric Traits for Personal Recognition Systems. In: Proceedings of International Conference on Biometric Authentication (ICBA). Hong Kong, LNCS 3072, 731-738.

JAIN, A. K. et al. (2004): Can soft biometric traits assist user recognition?. In: Proceedings of SPIE Biometric Technology for Human Identification. Orlando, FL, U.S.A., 5404, 561-572.

PATIL HEMANT, A. and BASU, T. K. (2004): Speech corpus for text/language independent speaker recognition in Indian languages. The National symposium on Morphology, Phonology and Language Engineering, SIMPLE04. A1-A4.

SCHEIDAT, T. and VIELHAUER, C. (2005): Fusion von biometrischen Verfahren zur Benutzerauthentifikation. In: P. Horster (Ed.), D-A-CH Security 2005 Bestandsaufnahme, Konzepte, Anwendungen, Perspektiven. 82-97.

SCHIMKE, S. et al. (2004): Cross Cultural Aspects of Biometrics. In: Proceedings of Biometrics: Challenges arising from Theory to Practice. 27-30.

TOMAI, C.I. et al. (2004): Group Discriminatory Power of Handwritten Characters. In: Proceedings of SPIE-ISET Electronic Imaging. 5296, 116-123.

VIELHAUER, C. et al. (2005): Finding Meta Data in Speech and Handwriting Biometrics. In: Proceedings of SPIE-IS\&T. 5681, 504-515.

VIELHAUER, C. (2006): Biometric User Authentication For IT Security: From Fundamentals to Handwriting. Springer, New York, U.S.A., to appear 2006. 